\title{
Erratum to: Characterization of the conserved phosphorylation site in the Aspergillus nidulans response regulator SrrA
}

\author{
Daisuke Hagiwara • Takeshi Mizuno • \\ Keietsu Abe
}

Published online: 12 April 2011

(C) Springer-Verlag 2011

\section{Erratum to: Curr Genet (2011) 57:103-114 \\ DOI 10.1007/s00294-010-0330-2}

In the original version, there were some overlaps in Fig. 1.

The corrected figure is produced in the following page.

The online version of the original article can be found under doi:10.1007/s00294-010-0330-2.

D. Hagiwara $\cdot$ K. Abe $(\bowtie)$

New Industry Creation Hatchery Center,

Tohoku University, 6-6-10 Aoba, Aramaki,

Aoba-ku, Sendai 980-8579, Japan

e-mail: kabe@biochem.tohoku.ac.jp

T. Mizuno

Laboratory of Molecular Microbiology,

School of Agriculture, Nagoya University,

Furocho, Chikusa-ku, Nagoya 464-8601, Japan

K. Abe

Laboratory of Applied Microbiology,

Department of Microbial Biotechnology,

Graduate School of Agricultural Science,

Tohoku University, 1-1 Amamiya,

Tsutsumi-dori, Sendai 981-8555, Japan

Present Address:

D. Hagiwara $(\bowtie)$

Department of Biological Sciences, Chuo University, 1-13-27 Kasuga, Bunkyo-ku, Tokyo 112-8551, Japan e-mail: dhagi@kc.chuo-u.ac.jp 


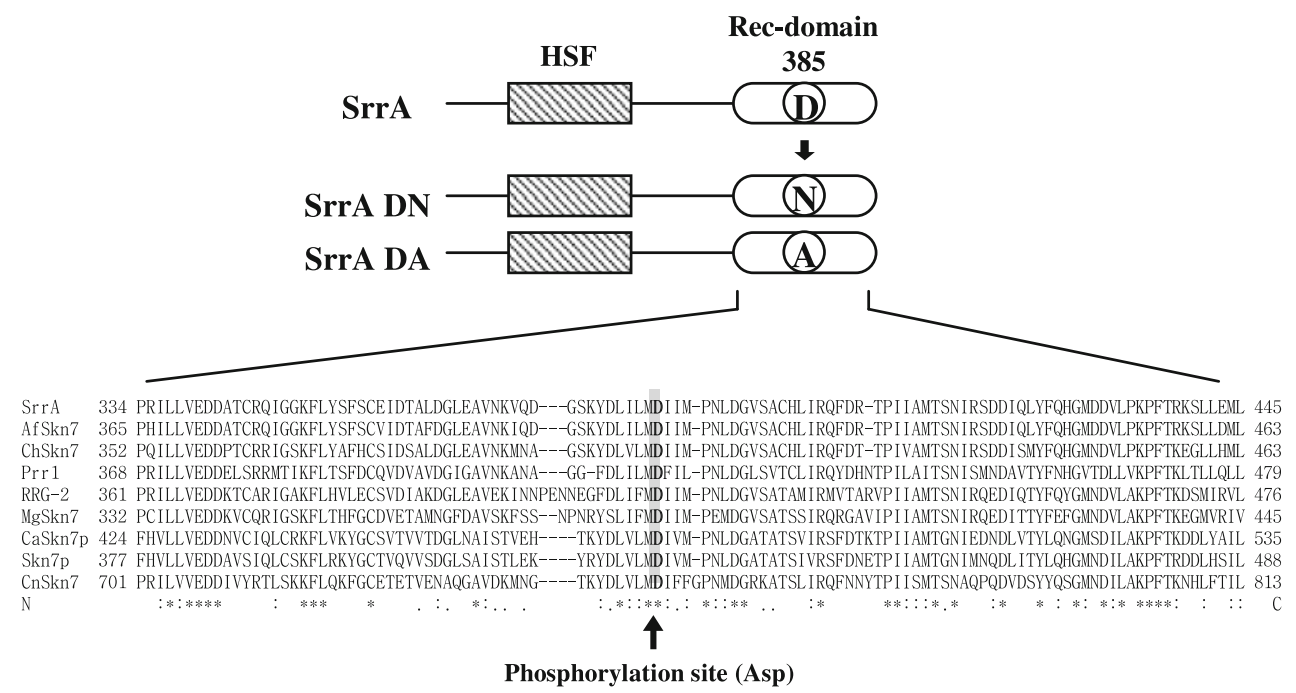

Fig. 1 Structure of SrrA protein and sequence alignment of the receiver domain. (Top) Schematic representation of the structure of SrrA and the substitution mutant constructs used in this work. The wildtype SrrA comprises two characteristic domains: a eukaryotic heat shock factor-type DNA-binding motif (HSF) and a receiver domain (Rec-domain) with an invariant aspartate residue (D385). In the SrrA variants SrrA DN and SrrA DA, the invariant aspartate was replaced with asparagine $(\mathrm{N})$ or alanine (A), respectively. (Bottom) The amino acid sequence of the receiver domain of SrrA was aligned with that of orthologs for other fungi (see descriptions in Table 1), using Clustal X. The employed sequences were obtained from SrrA (GenBank accession No. XP_661292), AfSkn7 (XP_001481472), ChSkn7 (BAI48020), Prr1 (CAB16301), RRG-2 (BAE93903), MoSkn7 (XP_360973), CaSkn7p (AAQ08008), Skn7p (NP_012 076), and CnSkn7 (AAX62808). The numbers shown at the ends of each sequence indicate the positions with respect to the initiation peptide. The predicted phosphorylation site (invariant aspartate residue) is indicated by bold characters and gray shading 